\title{
SEXO, DEMOCRACIA Y PODER POLÍTICO
}

\author{
ROSA COBO \\ Universidad de A Coruña
}

Parece existir consenso en el mundo de las ciencias sociales y políticas en torno a la idea de que estamos en un momento histórico de fuertes cambios sociales que están trastocando tanto nuestra visión del mundo como nuestras condiciones sociales de existencia. El cambio social es tan significativo que incluso nuestros modelos de investigación social no parecen tener la suficiente capacidad explicativa como para dar cuenta del nuevo mundo que se está gestando. Así, un nuevo paradigma de conocimiento -Castells lo denomina paradigma informacionalista- está dando respuesta a algunos cambios que están arrasando no sólo con viejas costumbres y creencias sino también con instituciones que ya no satisfacen las demandas y necesidades de este nuevo mundo global.

Estos cambios, marcados por las tecnologías informacionales, están produciendo nuevas estructuras sociales y nuevas estratificaciones en las que el reforzamiento de las políticas neoliberales se ve correspondido por la pérdida de espacio de la política y del estado. La globalización de las políticas neoliberales y el recorte global de las políticas sociales están precarizando la democracia. Por ello, no es de extrañar que uno de los debates más recurrentes en los últimos años en teoría política y teoría social sea el de la crisis de la política y de la democracia representativa. Los argumentos utilizados desde posiciones postmodernas, comunitaristas y multiculturales han criticado la incapacidad de la democracia para gestionar los intereses y las necesidades de distintas minorías y grupos sociales. De otro lado, desde posiciones universalistas radicales también se ha subrayado las dificultades de las democracias liberales para neutralizar desigualdades económicas y culturales. Unos y otros han concluido que la «base conceptual de la política contemporánea está en crisis» ${ }^{1}$. Una de esas desigualdades estructurales difícil de desactivar y que pone en cuestión la legitimidad de la democracia es la ausencia de las mujeres en los espacios de poder.

1. Scott, Joan: «La querelle de las mujeres a finales el siglo XX», New Left Review (Madrid), 3 (2000), p. 105. 
Lo cierto es que esta neoliberalización de la democracia está precarizando la vida de las mujeres e intensificando esa desigualdad estructural. En efecto, de un lado se está incrementando el trabajo gratuito femenino en el ámbito doméstico, pues las mujeres están asumiendo el coste del desplazamiento de los recortes sociales a la familia ${ }^{2}$; y de otro lado, la participación de las mujeres en el mercado laboral mundial va asociada a la precarización, pérdida de derechos y condiciones de trabajo propias del siglo XIX ${ }^{3}$. El resultado de todo ello es el incremento de la feminización de la pobreza.

En medio de esta reflexión sobre la crisis de la democracia representativa y los efectos de las políticas económicas neoliberales, las feministas han iniciado un nuevo debate en torno a la baja participación de mujeres en el poder político ${ }^{4}$. Y muchos datos ponen de manifiesto que el debate sobre la democracia paritaria va a ser una de las grandes discusiones políticas del siglo XXI. En todos los países del mundo en los que existe un movimiento feminista se está proponiendo un nuevo reparto de poder entre varones y mujeres.

El movimiento por la paridad surge en Francia a principios de la década de los noventa al mismo tiempo que se extiende por la Europa comunitaria ${ }^{5}$. La declaración de Atenas del año 1992 contiene una vindicación política de participación paritaria de las mujeres en el poder político y una exigencia de renegociación del contrato social. Las feministas europeas parten del supuesto de que el contrato social, pese a su formulación ética y política universal, se ha desarrollado a lo largo de la modernidad en la dirección de satisfacer las aspiraciones de ciudadanía de los varones. El feminismo europeo sostiene que la exclusión de las mujeres del contrato social exige la redefinición del mismo a fin de transformar las actuales democracias patriarcales en democracias más representativas y más legítimas ${ }^{6}$. Lo cierto es que los «críticos contra la paridad se están enfrentando a un movimiento político que está creciendo en Francia, liderado por una alianza inusual entre mujeres cuyas carreras se han desarrollado en el seno de partidos políticos, cargos electos y la administración pública» ${ }^{7}$.

El debate sobre la paridad está ganando cada vez más legitimación en las sociedades europeas, aún en medio de resistencias masculinas, a veces explícitas y a veces disfrazadas o enmascaradas detrás de otros debates que aparentemente

2. BAKER, Isabelle: «Dotar de género a la reforma de la política macroeconómica en la era de la reestructuración y el ajuste global», en Cristina Carrasco (ed.): Mujeres y economía, Barcelona, Icaria, 1999, pp. 267-268.

3. Beneria, Lourdes: "Mercados globales, género y el hombre de Davos», en Cristina Carrasco (ed.): Op. cit., p. 400.

4. MiYares, Alicia: Democracia feminista, Madrid, Cátedra, col. Feminismos, 2003, pp. 175-211.

5. Gaspard, Françoise; Le Gall, Anne y Ervan-Schreiber, Claude: Au pouvoir citoyennes! Liberté, égalité, parité, Paris, Le Seuil, 1992. Ver también Collin, Françoise: «La parité: une autre démocratie pour la France?», Les Cahiers du Grif, 47 (1993).

6. Gallego, Maite y SubiRats, Marina: "Democracia paritaria: Recorrido histórico y planteamiento actual», en Paloma SaAvedra (dir.): Hacia una Democracia Paritaria, Madrid, CELEM, 1999, pp. 53-62.

7. Scotr, Joan: Op. cit., p. 100. 
nada tienen que ver con esta vindicación. La paridad es una propuesta política discutida porque ataca el núcleo básico de la democracia patriarcal al proponer una nueva distribución de poder entre varones y mujeres. El objetivo último de esta propuesta política es alterar la estructura patriarcal del poder. Tal y como señala Ana Rubio, la cuestión de la paridad no es un problema técnico sino político ${ }^{8}$. Lo que se discute no es sólo la confección de las listas electorales o la distribución de puestos en determinadas instituciones. Lo que está en juego con esta política son las relaciones de dominación y subordinación entre hombres y mujeres. Las políticas feministas de la paridad no están dirigidas sólo a transformar las leyes electorales o las constituciones: su objetivo es cambiar la estructura profunda de poder entre los géneros que subyace a las democracias liberales y que sitúa a las mujeres en una situación de permanente discriminación.

El concepto de paridad, y de democracia paritaria, se inscribe en lo que Celia Amorós denomina el género "vindicación» ${ }^{9}$ y su objetivo es irracionalizar el monopolio masculino del poder. La vindicación política de democracia paritaria se inscribe en la historia de las vindicaciones feministas a favor de la igualdad y desde ese punto de vista es la prolongación de la lucha por el voto del movimiento sufragista. La paridad plantea que el interés por lo público y lo político, y la tarea que se deriva de ese interés, debe recaer igualmente en varones y mujeres. La noción de democracia paritaria nace de la contradicción entre el aumento de mujeres en muchos de los ámbitos de la vida social y su ausencia de los espacios donde se votan las leyes y se toman decisiones que afectan al conjunto de la sociedad y muy particularmente a las vidas de las mujeres.

Ahora bien, tal y como señala Joan Scott, el aspecto más interesante de este debate sobre la crisis de la política contemporánea radica en que por primera vez se ha centrado en la relación entre sexo y poder ${ }^{10}$. Y también por primera vez el sector más numeroso del feminismo ha considerado que la conquista del poder político puede traducirse en alteraciones reales en la jerarquía de género. El feminismo cuestiona la legitimidad de una democracia en la que la mayoría de sus instituciones representativas excluyen a las mujeres, no legalmente pero sí de hecho. El feminismo que se inscribe en la tradición igualitaria sostiene que no hay democracia política legítima que excluya a la mitad de la población. Y por ello plantea la paridad como un proceso estratégico de lucha contra el monopolio masculino del poder.

Desde este punto de vista, hay que poner de manifiesto que el debate que plantea la vindicación de democracia paritaria no se acaba en la necesidad de cuotas paritarias de mujeres en las instituciones de representación del estado. $\bigcirc$, por lo menos, no puede reducirse sólo a eso, porque la baja representación de mujeres en el poder político no es un problema técnico. Es un problema político resultado de una larga historia de intencionada exclusión de las mujeres de

8. Rubio, Ana: Los derechos humanos de las mujeres. Un nuevo contrato social (en prensa).

9. Amorós, Celia: Tiempo de feminismo. Sobre feminismo, proyecto ilustrado y postmodernidad, Madrid, Cátedra, col. Feminismos, 1997, p. 56.

10. Scott, Joan: Op. cit., p. 105. 
todos aquellos espacios en los que se dirimen recursos económicos, políticos y culturales.

El poder político no está aislado del conjunto de la sociedad, más bien forma parte de un orden estructural y, en muy buena medida, está al servicio de la reproducción de los sistemas sociales. Este hecho condiciona decisivamente el debate teórico en torno a la democracia paritaria y en torno a las estrategias para concretar las políticas de la paridad. Para que las estrategias vindicativas de paridad puedan tener resultados a largo plazo y no se conviertan en medidas políticas coyunturales deben identificarse analíticamente las raíces de la subordinación y exclusión de las mujeres, para lo que es imprescindible entender que la inferioridad social de las mujeres está asentada sobre fenómenos estructurales profundos.

La idea que subyace tras el debate sobre la democracia paritaria es que su concreción puede suponer un paso adelante cualitativa y cuantitativamente en la emancipación de las mujeres. Pero, como señalábamos anteriormente, sólo podrían quebrar la reproducción de determinadas instancias masculinas si se tiene una visión histórica y causal de la baja presencia de mujeres en el poder político. Así y todo, hay que subrayar que la conquista de la paridad es una cuestión elemental de justicia para las mujeres, sean feministas o no lo sean quienes ocupen puestos de poder en las instituciones. Pero no es suficiente el conocimiento histórico y causal de la exclusión y opresión de las mujeres para que una medida política tenga éxito. También es necesario que el feminismo se dote de una estrategia política. En este sentido, si la lucha por la paridad se construye como una estrategia feminista, resultado de pactos políticos entre mujeres de distintos ámbitos políticos y espacios sociales, y se genera un movimiento social organizado en la sociedad civil orientado a apoyar la lucha por la paridad, entonces las políticas de la paridad pueden erosionar la hegemonía política masculina.

La paridad, como hemos señalado y volveremos a señalar más adelante, no es un problema de números y porcentajes sino un instrumento político orientado a socavar esa realidad estructural que es el patriarcado. La baja representación de las mujeres en el poder político es el resultado de un sistema hegemónico de los varones dirigido a apropiarse de la mayoría de los recursos políticos, económicos y culturales de las mujeres. Si la paridad se analiza sólo como porcentajes, nos privamos de visibilizar la estructura de dominación masculina que ha producido históricamente la exclusión de las mujeres del poder político. Sin este análisis, la vindicación de paridad se convierte en mera táctica y se vacía de contenido transformador y crítico. Dicho de otra forma, difícilmente se pueden modificar las relaciones asimétricas de poder entre los géneros si no se plantea la paridad como una estrategia feminista fundada en pactos políticos entre distintos grupos de mujeres.

\section{POLÍTICAS Y ESTRATEGIAS FEMINISTAS PARA EL SIGLO XXI}

Cuando el feminismo comienza a configurarse como discurso teórico y como práctica política en el siglo XVIII, como todos los recién creados planteamientos intelectuales y movimientos sociales críticos, carece de aparatos 
conceptuales y de estrategias políticas. Esto hará que el feminismo comience a aproximarse a otros pensamientos críticos que identificaron relaciones de dominación y subordinación en la sociedad, como, por ejemplo, el marxismo. No en vano se configurará una poderosa corriente de feminismo marxista. Pero el feminismo no sólo tomará prestados algunos análisis del marxismo para deslegitimar la dominación masculina, también acudirá al psicoanálisis con la pretensión de entender cómo se construyen desde el nacimiento los modelos masculino y femenino. Y este paradigma proporcionará algunas herramientas conceptuales para entender la formación de las normatividades masculina y femenina. Sin embargo, hay que señalar que esta relación entre el feminismo con el marxismo y el psicoanálisis, entre otros enfoques, ha sido tortuosa porque esos pensamientos intentaron que el feminismo renunciase a constituirse como paradigma teórico y movimiento político. El resultado es una importante disputa, plasmada en una bibliografía abundante, en la que el feminismo ha constituido su propio punto de vista articulado sobre la existencia de la opresión de las mujeres. Inicialmente el feminismo encontrará armas críticas en otros movimientos y paradigmas de pensamiento que no aceptan como dada la realidad social y este hecho hará que el feminismo identifique la opresión de las mujeres en clave de explotación económica y en clave de déficit de derechos civiles y políticos, como por ejemplo, el derecho al voto. Y de ahí también que, en muy buena medida, el feminismo entienda que la opresión de las mujeres tiene una poderosa dimensión económica y política y asuma, como explican Young y Fraser, el paradigma distributivo que tan lúcidamente había identificado el marxismo para los varones.

Sin embargo, a medida que el paradigma feminista se va construyendo en medio de las dificultades teóricas, académicas y políticas propias de un discurso y una práctica que aspira a desactivar un sistema de dominación como es el patriarcado, a partir de los años setenta el feminismo toma conciencia de que la opresión de las mujeres tiene muchos más rostros que aquellos que pueden satisfacer las vindicaciones distributivas. Las obras de Nancy Fraser, Iustitia Interrupta, e Iris Young, La justicia y las políticas de la diferencia ${ }^{11}$, entre otras, explican lo que ya estaba en muy buena medida en el feminismo de los años setenta, en Kate Millett y Sulamith Firestone: que la opresión de las mujeres tiene unas marcas mucho más extensas que la de la explotación económica y la exclusión política. Ahí comienza a hablarse de la sexualidad como otro de los núcleos de dominación patriarcal. Y la familia como una de las instituciones cruciales en las que se desarrolla la dominación de los varones sobre las mujeres. Por otra parte, se identifica el patriarcado como un sistema de dominación que se asienta sobre la estructuración de la sociedad en dos ámbitos, el público y el privado-doméstico, en los cuales se inscriben todas las poderosas instancias de hegemonía de

11. Fraser, Nancy: Iustitutia Interrupta. Reflexiones críticas desde la posición postsocialista, Santafé de Bogotá, Siglo del Hombre Editores, 1997 y Young, Iris Marion: La justicia y las políticas de la diferencia, Madrid, Cátedra, col. Feminismos, 2000. 
los varones sobre las mujeres. Se concluye que la opresión de las mujeres tiene tantas marcas que las políticas de distribución ya no son capaces de neutralizar los mecanismos de dominación patriarcal.

De ahí que se reflexione desde el feminismo sobre otras estrategias que vayan más allá de la distribución económica y de recursos y se propongan otras medidas relacionadas con el reconocimiento o con las llamadas políticas de la identidad. La postmodernidad se declara fervientemente a favor de políticas deconstructivas y parece que el pensamiento de la diferencia sexual reclama con igual fervor políticas de la identidad y del reconocimiento. Sin embargo, no parece plausible que la opresión de las mujeres pueda resolverse únicamente con una de estas políticas.

Young explica que el paradigma de la distribución no es capaz de dar cuenta y de plantear políticas adecuadas para neutralizar la opresión de las mujeres. Señala esta autora que el paradigma distributivo, tan deudor del marxismo y de la socialdemocracia, "proporciona un fructífero punto de partida, pero es al mismo tiempo demasiado acotado y demasiado general $»^{12}$, pues se centra en la distribución económica pero no tiene en cuenta estructuras opresivas para las mujeres como la familia o esa división de la que hablábamos anteriormente, que es la separación de la sociedad en un ámbito privado y otro público-político. Subraya Iris Young que el paradigma distributivo enfatiza la distribución de bienestar, ingresos y posiciones, pero tiende a ocultar las estructuras y prácticas, las reglas y normas que las guían. Y no sólo eso, asimismo señala que este paradigma silencia el lenguaje y los símbolos que median las interacciones sociales dentro de dichas estructuras y prácticas en instituciones tales como el estado, la familia, la sociedad civil o el trabajo ${ }^{13}$. Concluye esta autora indicando que una teoría de la justicia no puede agotarse en un paradigma distributivo y que tiene que incorporar los conceptos de dominación y opresión si quiere dar cuenta cabal de la posición de las mujeres en las sociedades patriarcales. Conceptos, por otra parte, que el feminismo hace ya mucho tiempo que ha incorporado como cruciales a su arsenal político y conceptual para entender la dominación masculina.

Hay que señalar, sin embargo, que el paradigma distributivo, que buena parte del feminismo hizo suyo, no implicó el silenciamiento u ocultación del sistema de dominio patriarcal. Más bien, estas políticas se gestaron cuando el feminismo estaba implicado en la defensa de la autonomía económica de las mujeres y su acceso al mercado laboral. Y parece que la vindicación de políticas redistributivas son una condición de posibilidad para después plantear políticas de reconocimiento.

Por su parte, Nancy Fraser también explica que la política de la redistribución está orientada a colectividades que se pueden identificar en la estructura económica de la sociedad. Por ejemplo, el concepto de clase social del marxis- 
mo está inscrito en una diferenciación social arraigada en la estructura político-económica de la sociedad. Y, desde luego, si tomamos en consideración la variable "género" podemos observar que desde todos los puntos de vista las mujeres constituyen un colectivo con problemas de explotación económica en todos los sentidos. Más aún, los procesos de globalización neoliberal están empobreciendo paulatinamente a las mujeres en casi todos los países del mundo y se está haciendo una realidad cada vez más rotunda la llamada «feminización de la pobreza».

Ahora bien, las sociedades patriarcales no sólo se basan en la explotación económica de las mujeres. Hay otros muchos elementos que ponen de manifiesto que la opresión de las mujeres es multidimensional. De ahí que Young reclame la incorporación de los conceptos de dominación y opresión a la teoría de la justicia y que Fraser entienda el reconocimiento como un concepto con capacidad de explicar otros datos de opresión de las mujeres y como una política con posibilidades de desactivar algunos de los mecanismos de la dominación. Esta autora considera que además de la injusticia económica existe otra injusticia cultural o simbólica. Esta injusticia, en su opinión, está arraigada en los patrones sociales de representación, interpretación y comunicación e incluye la dominación cultural, el no reconocimiento y el irrespeto. La falta de reconocimiento se produce cuando las mujeres son invisibilizadas a través de prácticas representativas, interpretativas y comunicativas de la propia cultura. Y el irrespeto cuando se menosprecian con representaciones culturales públicas estereotipadas o en las propias interacciones cotidianas.

Nancy Fraser explica con toda razón que estas dos injusticias, la relacionada con la injusticia económica y la producida por la falta de reconocimiento, son distinciones analíticas pues en la realidad siempre aparecen mezcladas. Y es que habitualmente las injusticias económicas suelen tener dimensiones culturales y las culturales, a su vez, están vinculadas a explotaciones económicas. Esto le lleva a la autora a señalar que las mujeres están inscritas en esta doble dominación y que por ello mismo constituyen una comunidad bivalente.

Sin embargo, una vez que Fraser ha identificado estas dos variantes de dominación de las que las mujeres son objeto, se pregunta acerca de las políticas necesarias y útiles para combatir ambas discriminaciones. Y aquí es dónde ella señala que las políticas redistributivas por lógica deben desembocar en la desaparición del grupo dominado mientras que las políticas de la identidad o del reconocimiento suelen reforzar al grupo en cuestión. Explica que las injusticias económicas requieren reestructuraciones políticas y sociales en términos de ingresos, reorganización de la división del trabajo y cambios en los procedimientos de toma de decisiones. De otro lado, la solución para la injusticia cultural es algún tipo de cambio cultural o simbólico orientado a la transformación de los patrones sociales de representación, interpretación y comunicación con el fin de lograr cambios en la autoconciencia de todos los individuos de la sociedad. Señala que las exigencias de redistribución suelen ir dirigidas a la abolición de los mecanismos económicos que producen desigualdad y que en consecuencia suelen acabar en el fin de la diferenciación de los grupos explotados. Por el 
contrario, las exigencias de reconocimiento suelen enfatizar la especificidad de los grupos dominados y la afirmación de su valor como tales. En algunos casos, incluso esta política de reconocimiento puede llegar a visibilizar grupos que antes no tenían una existencia cultural ${ }^{14}$.

Diversos autores y autoras han hecho notar que en este nuevo siglo en el que acabamos de entrar se está proponiendo un nuevo imaginario político ${ }^{15} \mathrm{y}$ ciertamente se están observando indicios claros de que los valores acomodados en el centro simbólico de la sociedad están cambiando. En este nuevo imaginario político están comenzando a perfilarse tres tipos de políticas dirigidas a satisfacer demandas relacionadas con el déficit de derechos y de recursos de distintos grupos sociales y las mismas están vinculadas a tres paradigmas teóricos:

Las políticas del reconocimiento y de la identidad tienen un fuerte vínculo con el feminismo de la diferencia y otros planteamientos que giran en torno a la diferencia sexual. Todas estas políticas buscan reforzar la identidad de grupo, en este caso la identidad de las mujeres, y la idea fuerte que las vertebra es la ontologización de la identidad de género. Para el paradigma de la diferencia sexual la identidad es una liberación, pues en la preservación de la identidad, individual y colectiva, aún al precio de su esencialización, está la garantía de la recuperación y reconocimiento de la auténtica naturaleza de las mujeres.

Sin embargo, las politicas postmodernas o deconstructivas están emparentadas con la postmodernidad y su objetivo es deconstruir las identidades de grupo en el mismo instante en que se consoliden, pues esta consolidación pronto deviene normativa y coactiva con sus miembros. Lo que subyace en la postmodernidad es la desestabilización de la identidad de los grupos y minorías sociales. La idea de fondo es la deconstrucción de las identidades. Para la postmodernidad en general y para el feminismo postmoderno en particular la identidad es una prisión. Librarse de esa identidad es la condición de posibilidad de su liberación y un acto de resistencia frente a la dominación.

Frente a las políticas del reconocimiento y las políticas deconstructivas o postmodernas, los feminismos de la igualdad están proponiendo desde hace diez años políticas de la paridad. Estas políticas, que asumen aspectos de las propuestas del reconocimiento y de la identidad y, a su vez, debido a su marcado antiesencialismo coinciden con las políticas deconstructivas, son políticas de la vindicación que apuestan por el viejo paradigma redistributivo pero que entienden que es insuficiente. (Sin embargo, hay que precisar que no se pueden subestimar las políticas de redistribución de recursos económicos y políticos en momentos de exaltación de las políticas neoliberales y de creciente feminización de la pobreza).

Las políticas de la vindicación y de la paridad no creen que la identidad es una liberación ni tampoco una prisión. No proponen exclusivamente ni su on-

14. Fraser, Nancy: Op. cit. Véase el capítulo I: «Redistribución y reconocimiento», pp. 17-92.

15. QuesadA, Fernando: "Hacia un nuevo imaginario político», en VV.AA., Cambio de paradigma en la Filosofía Política, Madrid, Fundación Juan March, 2001, pp. 19-61. 
tologización ni su deconstrucción. Sostienen que todo grupo necesita dotarse de una identidad política provisional, contingente y antiesencialista para actuar como un actor político. Pero, al mismo tiempo, señalan que el horizonte es la desaparición de esa identidad colectiva y su sustitución por el reino de las individualidades. La idea de fondo de las filosofías de la igualdad es la desaparición del género como una realidad social que se ha traducido históricamente en fuente de opresión para las mujeres.

\section{LAS MUJERES COMO SUJETO POLÍTICO COLECTIVO EN UN MUNDO GLOBAL}

En el pensamiento de la diferencia sexual subyace la idea de que las mujeres no son tanto un colectivo oprimido como 'otro' colectivo diferente del que forman los varones como género. Por el contrario, el pensamiento de la igualdad arranca de la idea de que las mujeres forman un colectivo discriminado e inscrito en un sistema de dominación que refuerza y reproduce la hegemonía de los varones y la inferioridad de las mujeres. La médula del planteamiento de la igualdad es, tal y como señala Amorós, la vindicación, es decir, la exigencia de que se apliquen a las mujeres los derechos civiles, sociales y políticos que se aplican a los varones. Y que se resumen en el uso paritariamente compartido de todo tipo de recursos, desde los económicos y políticos hasta los de autoridad y culturales. Sólo la vindicación política puede detener ese insidioso mecanismo social que coloca a las mujeres en una posición de subordinación. La perspectiva de la igualdad reivindica la radicalización de la ciudadanía y de la democracia para las mujeres: tanta ciudadanía y tanta democracia como sean necesarias para que las mujeres se constituyan en sujetos.

Sin embargo, el núcleo del planteamiento de la diferencia sexual es el rechazo de la vindicación, pues, como afirma Irigaray, hay que poner de manifiesto la singularidad sexual de las mujeres y su necesidad de articular un "singular propio» ${ }^{16}$. Mientras que el feminismo de la diferencia enfatiza positivamente la diferencia entre varones y mujeres, el feminismo postmoderno radicaliza el concepto de diferencia y lo extiende al colectivo de mujeres hasta hacer imposible la construcción del sujeto político colectivo «mujeres». El feminismo postmoderno niega el concepto de "mujeres», así como las nociones de género y patriarcado, pues parte de la idea de que esas abstracciones, tanto normativa como organizativamente, son muy coactivas para cada mujer. La conclusión es que los planteamientos de la diferencia siempre están al borde de ontologizar o esencializar (cuando no lo ontologizan por completo) el género con el consiguiente peligro de la ocultación de su carácter históricamente construido, mientras que los planteamientos postmodernos realizan la operación inversa, es decir, radicalizan la noción de diferencia individual hasta el punto de obstaculizar seriamente la posibilidad de construir un marco normativo para el feminis-

16. Irigaray, Luce: J'aime a toi: Esquisse d'une felicité dans l'histoire, Paris, Grasset et Frasquelle, 1992 , pp. 84-85. 
mo y un movimiento social estructurado. Las consecuencias políticas de estos planteamientos de la igualdad, de la diferencia sexual y de la postmodernidad difieren notablemente, sobre todo en la propuesta de las mujeres como un genérico emancipador y como un actor político con capacidad de intervención social y de negociación política en el contexto de sociedades patriarcales.

Hay un debate abierto en el seno del feminismo y de las ciencias sociales en general acerca de si las mujeres constituyen una categoría social como lo constituyen, por ejemplo, la etnia, la raza o la clase. La pregunta recurrente es si las mujeres forman una comunidad ${ }^{17}$. Para ir despejando dudas, hay que comenzar por señalar que las mujeres no son una minoría en un sentido numérico ni tampoco constituyen una cultura en sentido estricto. Las mujeres son la mitad de cada categoría social y de cada sociedad. Y no sólo eso: «la posición de las mujeres es un elemento estructural que determina el carácter de toda cultura» ${ }^{18}$. Por todo ello, puede decirse que las mujeres forman un colectivo cualitativa y cuantitativamente distinto a los ya nombrados. Su singularidad está relacionada con su presencia en todos los grupos sociales. Ahora bien, el hecho de que las mujeres no formen una categoría social como la clase o la etnia no significa que no puedan constituirse como un grupo social con vínculos de solidaridad.

Desde este debate, se pregunta Joan Scott si «existe un 'interés de las mujeres' que atraviese las barreras de clase, etnicidad y raza y, si así fuera, en qué consiste ${ }^{19}$. Susan Moller Okin reflexiona sobre esta cuestión explicando que el sexismo es una forma identificable de opresión, muchos de cuyos efectos son percibidos por las mujeres con independencia de la raza, clase o cultura a la que pertenezcan. Utilizando investigaciones empíricas sobre el trabajo de las mujeres en los países pobres, concluye que las condiciones de las mujeres del Tercer Mundo son «similares, pero más agudas y acentuadas» que las de los países ricos ${ }^{20}$.

Las mujeres constituyen un colectivo marginado y subordinado en todas y cada una de las sociedades existentes. Su pertenencia, sea voluntaria o asignada, a grupos que ostentan una posición dominante en la sociedad no las exime de la subordinación a los varones en el seno del grupo. Este hecho, la discriminación de género, constituye el fundamento de su identidad como colectivo. Ahora bien, ¿si en los colectivos étnicos o raciales la multiplicidad de sus diferencias internas -económicas, culturales, de género, de preferencia sexual o de estatus, entre otras- no son un obstáculo para que se les conceptualice como una minoría, por qué esas mismas diferencias no son suficientes para que las mujeres sean consideradas un grupo social? La desigualdad de las mujeres no es casual ni aleatoria. Por el contrario, se atiene a datos constantes que singularizan a las

17. Osborne, Raquel: «¿Son las mujeres una minoría?», Isegoría (Madrid), 14 (1996), pp. 79-93.

18. Balibar, Etienne: "Ambiguous Universality", Differences, 7-1 (1995), pp. 67-68. Citado en ScotT, Joan: Op. cit, p. 108.

19. ScotT, Joan: Op. cit., p. 104.

20. Okin, Susan Moller: «Desigualdad de género y diferencias culturales», en Carme Castells (comp.): Perspectivas feministas en teoría política, Barcelona, Paidós, 1996, pp. 190-194. 
mujeres frente a otros colectivos. Largos siglos sometidas a una socialización generizada que se ha concretado socialmente en el ejercicio de roles (esposa, madre, cuidadora, guardiana de las esencias culturales, entre otros), cuyo contenido las apartaba de lo público y de lo político y las confinaba al territorio de los cuidados, de los afectos y del trabajo gratuito, ha sido suficiente para que las sociedades patriarcales se hayan ido configurando lentamente como sociedades estratificadas desde del punto de vista del género.

Ahora bien, la precariedad es un rasgo inherente a los grupos sociales, como lo es a cualquier otra construcción social. Por ello, para neutralizar esta precariedad y garantizar su permanencia y reproducción social es necesaria la construcción de una identidad política que sea el resultado de una estructura organizativa y normativa, aunque ambas sean de mínimos. Los grupos sociales que se autocomprenden a sí mismos como colectivos discriminados se dotan de una identidad política, pues no hay grupo social sin identidad. Todo colectivo que aspira a convertirse en un actor social debe construirse una identidad política. Joan Scott explica muy bien que «la cuestión es que las mujeres son políticamente eficaces únicamente cuando se movilizan como categoría social» ${ }^{21}$.

El feminismo de tradición igualitaria considera la construcción de una identidad feminista como la condición de posibilidad para que las mujeres se constituyan en sujetos y para articular colectivamente la lucha política. Esta identidad, lejos de basarse en esencias u ontologías, como señala Amorós, es crítico-reflexiva respecto a la identidad femenina ${ }^{22}$. El feminismo de la igualdad ha señalado en numerosas ocasiones que no hay una identidad esencial femenina ni tampoco una unidad original y previa que recuperar. El fundamento en la construcción de una identidad feminista es que todas las mujeres comparten inicialmente una situación de discriminación. La identidad debe ser entendida instrumentalmente como el fundamento de la lucha contra la opresión, pero nunca como el enquistamiento en la diferencia o la exaltación de una esencia. Sin identidad colectiva no hay movimiento social, ni posibilidades de combatir la infrarrepresentación política ni económica, ni un discurso ideológico con posibilidades de ganar hegemonía política. La identidad es sobre todo el resultado de una elección política.

Puede parecer paradójico, pero un movimiento emancipador y vindicativo tiene que construirse una identidad contingente, afirmarla hasta que se conquisten los derechos de los individuos de ese colectivo y, al mismo tiempo, tiene que negar ontológicamente esa identidad si aspira a la realización de la universalidad $^{23}$. Y es que un sujeto político colectivo es una noción política y no

21. ScotT, Joan: Op. cit., p. 110.

22. Esta idea ha sido señalada por Celia Amorós en las sesiones de trabajo del proyecto «Feminismo, Ilustración y Posmodernidad» de la Universidad Complutense de Madrid que dirigió entre 1997-2000.

23. Balibar, Etienne: Op. cit., pp. 67-68. Citado por Scott, Joan: Op. cit., p. 108. 
un dato biológico o étnico ${ }^{24}$. Por ello, el rasgo de esta identidad es su carácter provisional y contingente. La afirmación de la identidad colectiva es un paso necesario en la constitución de las mujeres como sujetos individuales. Amelia Valcárcel explica que la construcción de la individualidad requiere de la formación de un «nosotras»: "Cualquier movimiento que se plantee cambiar determinados rasgos de la realidad política y social ha de educir un nosotros al que dotar de rasgos de legitimidad y excelencia. Paradójicamente construir la individualidad no es una tarea individual, sino colectiva ${ }^{25}$.

Este proceso es complicado y tiene por lo menos dos fases (que no transcurren tanto cronológica como simultáneamente): primero hay que dotarse de una identidad política emancipadora orientada al desmantelamiento de la identidad normativa asignada por el patriarcado a las mujeres (esposas, madres, cuidadoras...); y después, al tiempo que se desactiva la identidad impuesta patriarcalmente hay que negar aquella otra identidad política construida para combatir las jerarquías patriarcales. Dicho de otra forma, hay que evitar los peligros de la ontologización de la identidad de género, pues la naturalización de la identidad de un grupo social aleja a los miembros de dicho colectivo de la conquista de la individualidad y los devuelve otra vez al territorio de la uniformidad social de la que se quieren despegar. Y sólo entonces, cuando la codificación genérica se vuelve fluida puede constituirse un caldo de cultivo idóneo para el florecimiento de una nueva cultura del sujeto ${ }^{26}$.

Todo grupo oprimido carga con una identidad heterodesignada por quien se beneficia de esa opresión, en este caso el patriarcado, y ese mismo grupo si aspira a deshacerse de esa opresión tiene que construir una identidad de resistencia que sea capaz de transformarse después en lo que Castells denomina una «identidad proyecto». La «identidad proyecto» se produce «cuando los actores sociales, basándose en los materiales culturales de que disponen, construyen una nueva identidad que redefine su postura en la sociedad y, al hacerlo, buscan la transformación de toda la estructura social» ${ }^{27}$.

La identidad de género cuando se dota de un proyecto político que tiene la vista puesta en el fin de su opresión se convierte en un sujeto político. Lidia Cirillo explica que «ningún grupo puede unir a sus miembros en una relación de solidaridad, ser consciente de la naturaleza de sus necesidades y moverse en una dirección y no en otra, si no es capaz de expresar de alguna forma quién es» ${ }^{28}$. El "quién es» se traduce políticamente en la "identidad-proyecto»: "La identidad de un sujeto político de liberación es su identidad social, étnica, nacional, de color o de género, a la que se añade un proyecto» ${ }^{29}$.

24. CIRILLo, Lidia: Mejor huérfanas: por una crítica feminista al pensamiento de la diferencia, Barcelona, Anthropos, 2002, p. 33.

25. ValCÁrCel, Amelia: La política de las mujeres, Madrid, Cátedra, col. Feminismos, 1997, p. 80.

26. Amorós, Celia: Tiempo de feminismo..., op. cit. Véase el capítulo I: «Por un sujeto verosímil».

27. Castells, Manuel: La era de la información. Economía, sociedad y cultura. Vol. 2. El poder de la identidad, Madrid, Alianza, 1997, p. 30.

28. Cirillo, Lidia: Op. cit., p. 110.

29. Ibíd., p. 114. 
La existencia del feminismo está ligada tanto a la contribución de una alternativa política que contemple el fin de la subordinación y la exclusión, como a la desactivación de las identidades normativas de las mujeres y pacientemente construidas por los sistemas hegemónicos masculinos. La utilidad del feminismo está vinculada al debilitamiento de la normatividad femenina asignada por el patriarcado y a la creación de diversas normatividades menos rígidas y coactivas para las mujeres. Las identidades se construyen a lo largo de procesos históricos complejos. Entre las condiciones de la existencia marcadas por las diferencias de cuerpo, clase o color y la capacidad de convertirse en sujeto político se encuentra el puente indispensable de la identidad ${ }^{30}$.

La construcción de sociedades democráticas más justas, libres e igualitarias pasa necesariamente por la desactivación de una normatividad femenina que obstaculiza a las mujeres en su autoconstrucción como sujetos, privándolas de aquellos recursos (políticos, económicos, culturales o de autonomía personal) que hacen posible que un sujeto pueda actuar como tal. Entre la ampliación de la democracia y la ampliación de la ciudadanía hay una relación de necesidad y cualquier redefinición de la ciudadanía orientada a la ampliación de los derechos no puede ignorar una construcción normativa de lo femenino que restringe los derechos civiles, políticos y sociales de la mitad de la sociedad. Desde este punto de vista, la vindicación de democracia paritaria ensancha los derechos políticos de las mujeres y por ello añade legitimidad política y dimensión ética a las actuales democracias. 